\title{
A prospective study of one hundred cases of Amoebic liver abscess in a secondary care hospital of Delhi
}

\author{
Yogesh Kushwaha, Rajiv Kapil, Sarbjeet Khurana
}

Yogesh Kushwaha' ${ }^{1}$,Rajiv
Kapil' $^{2}$ Sarbjeet Khurana

1 Department of Medicine, Lal Bahadur Shastri Hospital, Delhi,INDIA.

${ }^{2}$ Department of Medicine, Lal Bahadur Shastri Hospital, Delhi,INDIA.

${ }^{3}$ Department of Epidemiology, Institute of Human Behaviour \& Allied Sciences (IHBAS), Delhi, INDIA.

\section{Correspondence}

Sarbjeet Khurana, Associate Professor \&HOD, Department of Epidemiology, Institute of Human Behaviour \& Allied Sciences (IHBAS), Delhi, INDIA.

Email: sarbjeetkh@gmail.com

\section{History}

- Submission Date: 09-02-16.

- Review completed: 21-05-16;

- Accepted Date: 22-06-16.

DOI : 10.5530/ijmedph.2016.2.6

Article Available online http://www.ijmedph.org/v6/i2

Copyright

(C) 2016 Phcog. Net. This is an open-access article distributed under the terms of the Creative Commons Attribution 4.0 International license.

\begin{abstract}
:
Background: Amoebic liver abscess (ALA), which is caused by Entamoeba histolytica a protozoa is common and serious extra-intestinal manifestation of amoebiasis. ALA is prevalent in areas of poor sanitation and hygiene. It has varied clinical presentations and complications. This study highlights the recent clinical trends of ALA.
\end{abstract}

Material and Methods: This study was a hospital based prospective observational study. All patients admitted from March to December 2011 with the diagnosis of ALA were included. Demographic and clinical history was recorded and the systematic examination was done to understand disease pattern. Clinical diagnosis was supported and confirmed by ultrasound abdomen and positive amoebic serology. The outcome of the patient was recorded at discharge. Data was analysed using SPSS 17.

Results: Out of total 100 ALA Patients, $72 \%$ were from urban slums, $88 \%$ were uneducated and belonged to low socioeconomic status. $76 \%$ were alcoholic, $52 \%$ patients were in age group of 21-40 yrs with male preponderance (89\%). Commonest presenting symptom was pain in right hypochondrium (94\%)while jaundice was noticed in only $4 \%$. Mean duration of symptoms before hospitalization was 10 days. Personal \& family history was not contributory. Hepatomeagly was present in 52\%. Mean TLC - 13694, Bilirubin - $1.27 \mathrm{mg} \%$. Serum Alkaline Phosphatase - $698 \mathrm{IU}$. X ray showed raised right diaphragm in $50 \%$ cases. On USG Examination 7\% located in left lobe of liver and $84 \%$ were more than $5 \mathrm{~cm}$ in size. Multiple abscesses in $21 \%$. Amoebic Serology was positive in all patients. Mean duration of hospitalization was 7 days. Management was done with metronidazole, antibiotics and/or percutaneous aspiration. Rupture occurred in 10\%. One patient died of DIC.

Conclusion: ALA is a medically treatable common infection prevalent in developing countries due to lack of sanitation and hygiene. Health education and adequate sanitation are imperative in preventing amoebic infections.

Key words: Amoebiasis, Amoebic liver abscess, Entamoeba histolytica, Hepatic abscess, liver abscess, Percutaneous aspiration.

\section{INTRODUCTION}

Amoebic liver abscess (ALA), which is caused by "Entamoeba Histolytica" a protozoa, is common and serious extra-intestinal manifestation of amoebiasis. Fedor Aleksandrovich suggested in 1875 that amoebic liver abscess is linked to amoeba. ${ }^{1}$ This disease is a major cause of morbidity and mortality in developing countries. It has been reported to be a major problem in India. ${ }^{2,3}$ ALA is known to be a disease prevalent in areas of poor sanitation and hygiene. According to WHO amoebiasis is prevalent in all developing countries and at times its prevalence reaches upto $50 \%$ of general population. It has varied clinical presentations and complications. This study was done to evaluate and investigate into the recent clinical trends of ALA.

\section{MATERIALS AND METHODS}

The present study was done at the Department of medicine of a secondary care Delhi Government hospital from March to December 2011. It was a prospective observational study. Informed and written consent was obtained from all the patients.

A total of 100 adult (age $>12$ yrs) patients with diagnosis of ALA were included in study. Diagnosis of ALA was made by clinical symptoms supported by ultrasonogrphy and confirmed by amoebic serology. All patients were treated with Metronidazole $2.4 \mathrm{gm}(40 \mathrm{mg} / \mathrm{kg} / \mathrm{d})$ for minimum 10 days \pm antibiotics \pm percutaneous aspiration followed by Diloxanate furoate $500 \mathrm{mg}$ thrice a day for 10 days. Demographic and clinical data was recorded in a pre defined proforma. Clinical history, systemic examination and other data were analysed to understand
Cite this article : Kushwaha Y, Kapil R, Khurana S. A prospective study of one hundred cases of Amoebic liver abscess in a secondary care hospital of Delhi. Int. J. Med. Public Health, 2016; 6(2):84-87. 
the disease pattern. The outcome of the patients was recorded at discharge. Data was analysed using SPSS 17.

\section{RESULTS}

The demographic, clinical features and laboratory data of all 100 patients admitted with diagnosis of ALA in our study were analysed and findings are as follows:

\section{Demographic Data}

The age of the study subjects varied from minimum 12 yrs to maximum 76 yrs with an average of 37.42 yrs. Maximum (52\%) subjects with ALA were in the age group of 21-40 yrs (Table 1). males were affected much more than females with male: female ratio of 9:1.

In the present study $72 \%$ patients were from urban slums, $19 \%$ from urban and $9 \%$ from rural area of Delhi (Figure 1). $88 \%$ cases were uneducated and majority belonged to the low socioeconomic status.76\% of subjects had significant history of alcohol consumption.

\section{Clinical profile}

Though the symptoms of ALA are non specific, but the main clinical manifestations were pain in right hypochondrium (94\%), weakness $(83 \%)$, fever (82\%), anorexia (73\%), vomiting (25\%), cough (24\%) and weight loss in in $22 \%$ of the study subjects. (Table 2 ).

Pain abdomen was dull aching to pleuritic in nature with radiation to the back, epigastrium or right shoulder. Fever was reported in $82 \%$ cases. Other symptoms reported were nausea, vomiting, cough, pedal oedema etc. $9 \%$ cases had history of diarrhoea and $4 \%$ had jaundice (Table 2 ). Mean duration of symptoms before admission was 10 days ranging from 2days to 45 days.

Table 1: Age \& sex Distribution of the study subjects

\begin{tabular}{ccc}
\hline Age Group (yrs) & $\begin{array}{c}\text { Sex(no.) } \\
\text { Male Female }\end{array}$ & Total (\%) \\
\hline$<20$ & 0903 & $12(12 \%)$ \\
$21-40$ & 4804 & $52(52 \%)$ \\
$41-60$ & 2503 & $28(28 \%)$ \\
$>60$ & 0701 & $8(8 \%)$ \\
Total & 8911 & 100 \\
\hline
\end{tabular}

Table 2: Clinical Symptoms and laboratory data on the day of admission

\begin{tabular}{cc}
\hline Symptoms & $\%$ of subjects \\
\hline Abdominal pain & $94 \%$ \\
Weakness & $83 \%$ \\
Fever & $82 \%$ \\
Anorexia & $73 \%$ \\
Nausea & $37 \%$ \\
Vomiting & $25 \%$ \\
Cough & $24 \%$ \\
Weight loss & $22 \%$ \\
Breathlessness & $16 \%$ \\
Diarrhoea & $9 \%$ \\
Pedal edema & $5 \%$ \\
Jaundice & $4 \%$ \\
Laboratory Tests & Mean (range) \\
Leukocyte Count & $13694.2\left(5900-35200 \mathrm{~mm}^{3)}\right.$ \\
S. bilirubin & $1.2(0.5-9.5 \mathrm{mg} \%)$ \\
SGPT & $67.7(11-834 \mathrm{U} / \mathrm{L})$ \\
SAP & $697(82-2850 \mathrm{U} / \mathrm{L})$ \\
\hline
\end{tabular}

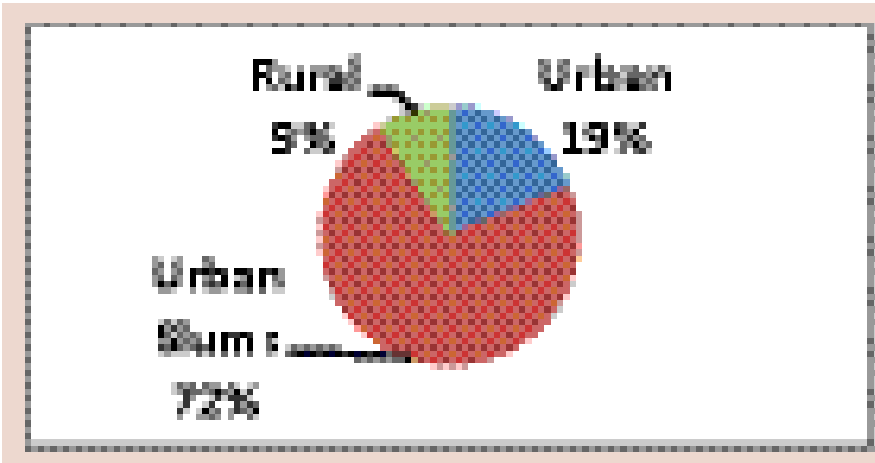

Figure 1: Distribution of Habitat of the study subjects

\section{Laboratory and radiological profile}

Leukocytosis (mean TLC -13694) with maximum 35200/ $\mathrm{mm}^{3}$ and elevated Serum Alkaline Phosphatase (mean: 698 U/L ranging from 82 to $2850 \mathrm{U} / \mathrm{L}$ ) were the commonest abnormal laboratory finding. The liver function tests were deranged i.e. S. bilirubin $>1.2 \mathrm{mg} \%$, SGPT and SGOT $>60$ IU/lt, (Table 2). There was no correlation among abscess size, serum bilirubin and Serum Alkaline Phosphatase. Serology tests for amoeba: Serology tests performed were IHA and IFA. All the cases had positive amoebic serology. (One of the inclusion criteria of the study design was to include only positive amoebic serology patients).

\section{Imaging studies}

$\mathrm{X}$ ray chest was abnormal in $55 \%$ cases. Most common finding in CXR was raised right hemidiaphragm (51\%). 3\% of the cases had right pleural effusion and $1 \%$ had a round right paratracheal opacity.

On ultrasonography in $94 \%$ of the subjects the abscess were single and $6 \%$ had bilateral abscess. Right lobe was affected predominantly in $86 \%$ of cases and $8 \%$ had only left lobe involvement. In $16 \%$ of the cases the abscess size was less than $5 \mathrm{~cm}$, and $84 \%$ had abscess of size $>5 \mathrm{~cm}$.

\section{Treatment}

All the patients were treated with Metronidazole $2.4 \mathrm{~g} / \mathrm{d}$ for a period of 10 days and then another drug patients were given Metronidazole $2.4 \mathrm{gm} / \mathrm{d}$ for 10 days followed by Diloxanate furoate was given in a dose of $500 \mathrm{mg}$ thrice a day for another 10 days with or without the antibiotics and /or percutaneous aspiration. Mean hospital stay was 7.89 days ranging from 3 days to 25 days. On aspiration pus colour varied from red, brown, and white, green to grey. Many times pus colour changes while aspirating from same patient from same cavity. Aspiration is a safe procedure and only one patient had bleeding into cavity that was managed conservatively. One patient died of sepsis and DIC.

\section{Complications}

Amoebic liver abscess is known to lead to various complications .55\% of the patients had elevated right hemidiaphragm. Pleural effusions, ascites and subhepatic collection were encountered in 3\% each. $2 \%$ had peritonitis and splenic abscess was also seen in $2 \%$ of the patients. One patient died of sepsis.

\section{DISCUSSION}

Amoebiasis is one of the common infections in the developing countries like India, tropical regions of Africa, Mexico, Brazil; as well as it is a rising infection in developed countries such as USA or European 
countries as a result of infected people visiting these countries or citizens of these countries visiting the endemic countries. ${ }^{(4,5,6)}$

The mean age of the study subjects in our present study was $37.4 \mathrm{yrs}$ and most of the patients were in the age group $20-40 \mathrm{yrs}$. This is comparable to various studies. $3,7,9,10$

Males were predominantly affected, and the Male: Female ratio was 9:1. Globally it has been observed that males are affected much more than females. ${ }^{3,7,8,10}$

A review of 8800 cases of ALA was conducted by Wells and Arguedas and they reported that males were predominantly affected and accounted for $86 \%$ of the cases. ${ }^{8}$

Various theories have been postulated to explain this gender difference but the exact reason is still not known. Researchers have tried to explain the alcohol consumption in men, ${ }^{11}$ hormonal influences in premenopausal women, ${ }^{12}$ protective effect of iron deficiency anaemia among menstruating women, ${ }^{13}$ as some of the reasons for males being affected more than females.

Majority of the study subjects were from urban slums, low socioeconomic status and were illiterate. Similar findings have been reported by Ramani et al. ${ }^{7}$ various studies worldwide have emphasized the role of lack of sanitation and poor hygienic conditions leading to oro-fecal transmission of amoebic infection.

In our study, $76 \%$ of the study subjects had history of alcoholism. Alcohol has been identified as a major predisposing factor in the pathogenesis of ALA. Hai et al. in his study reported that $85 \%$ of his study subjects had history of alcoholism and also concluded that there is a fivefold incidence of ALA among alcoholics. ${ }^{2}$ Ramani et al. had reported alcoholism in $64 \%$ of their study subjects,similar findings have been reported by various other researchers. ${ }^{7,14,15}$ The literature points towards an association between alcohol intake and ALA which could possibly be due to hepatic damage by alcohol which predisposes the amoebic invasion, compromised nutrition and decreased resistance of the body and thereby suppressed liver function or it could be contaminated liquor with amoeba. ${ }^{2}$

Clinically majority of patients presented with pain abdomen (94\%) and fever (82\%). $67-87 \%$ of patients with amoebic liver abscess had fever and $62-94 \%$ had pain abdomen. ${ }^{16}$ Wells and Arguedas reported that the most common clinical manifestations in the ALA patients are abdominal pain, reported in $92 \%$ of patients and fever in $90 \%$ of the patients. Ramani et al. and Viroj Wiwanitkit also reported similar results. ${ }^{7,17}$ In a study by Sharma et al, $94 \%$ of patients had fever and $90 \%$ had pain abdomen. ${ }^{18}$

Various studies have reported jaundice in $45-50 \%$ of the cases of amoebic liver abscess, ${ }^{1,19,20}$ whereas in our study the only $4 \%$ of the cases had jaundice. Sharma et al. reported in his study on ALA that only $12.7 \%$ of cases were suffering with jaundice. He concluded that probably because of the effective anti-amoebic therapy coupled with the invasive catheter drainage, jaundice is now less frequently seen in cases of ALA. ${ }^{18}$

Distinct findings on laboratory investigation in our study were leukocytosis, raised SAP, and a positive amoebic serology. Similar results have been reported in various other studies. ${ }^{7,8,18}$

Raised right hemidiaphragm or pulmonary complications can be of diagnostic value on CXR. 51\% of the patients had raised right hemidiaphragm and $3 \%$ had pleural effusion. Similar results have been observed by Wells and Arguedas. ${ }^{8}$

ALA can be well documented with USG which is readily available, non invasive, safe and affordable. On USG Examination of ALA cases in our study majority (86\%) abscess were located in right lobe of liver and were single. Similar findings have been reported by Nari et al, Sharma and Ahuja 2003 reported that right lobe of the liver is usually affected and is predominantly a solitary lesion in $30-70 \%$ of cases. ${ }^{21}$
$55 \%$ of the patients had pulmonary complications. Yoo $\mathrm{HM}$ and co-workers had reported that $56 \%$ of their study subjects of amoebic liver abscess had pulmonary complications. ${ }^{9}$ In our study out of those who had pulmonary complications the most common was elevated right hemidiaphragm (51\%). Ribaudo \& Ochsner from the USA reported diaphragmatic elevation in $71 \%$ of their series of 21 cases, while Nwafo \& Egbue from Nigeria reported a $31.8 \%$ incidence of the high right hemidiaphragm. ${ }^{21,22}$ Lyche et al. reported that $27 \%$ of his study subjects elevated right hemidiaphragm. ${ }^{23}$

Pleural effusion is also a common complication of ALA and usually occurs in the right lobe .3\% of our patients had pleural effusion. Sharma et al. has reported that $8.1 \%$ of the his patients had developed pleural effusion whereas it was observed to be $30 \%$ in a study by Lyche et al. ${ }^{24}$ In a study by Adebayo 0 Adeyemo and Adebimpe derounmu $(20.5 \%)$ of the patients of ALA had serous pleural effusion. ${ }^{25}$ Ascites was encountered in $3 \%$ of our patients. Tony and Martin reported that $1 \%$ of the patients developed ascites. ${ }^{26}$

Subhepatic collection was encountered in $3 \%$ of the patients in our study which is comparable with the results of the study by Yoo HM and co-workers. ${ }^{9}$

$2 \%$ of our study subjects had developed peritonitis. Sharma et al. in his study had reported that $4 \%$ of his patients of ALA had peritonitis. Wells et al. reported that in ALA, peritonitis is seen in 2 to $7 \%$ and often with left-sided abscesses. ${ }^{8}$ Similar results have been reported by other researchers. ${ }^{27-29}$

Splenic abscess in our study was observed in $2 \%$ of the patients. Occurrence of a splenic abscess is usually reported either after a rupture or due to the metastasis. ${ }^{30}$

In our study one patient (1\%) died of sepsis. Sharma et al. reported that the mortality rate in their study was $5.8 \%$ and further suggested that this was linked to an increased number of pigtails catheters that were inserted in the ALA cavity. Yoo HM and co-workers reported mortality in $3 \%$ of the study subjects of ALA and concluded that the mortality is much less in ALA as compared to the pyogenic abscesses which was as high as $17 \%$ in his study. ${ }^{9}$ In several studies of ALA, the overall mortality rate ranges from $2-15 \% .{ }^{1}$ ALA responds well to Metronidazole and mortality is low if treated early.

\section{CONCLUSION}

ALA is a medically treatable common infection prevalent in developing countries due to lack of sanitation and hygiene which are inherently linked with the disease causation. Hence the health policy should be directed towards improving the factors of health education and adequate sanitation which are imperative in preventing the amoebic infections.

\section{ACKNOWLEDGEMENT}

We acknowledge and thank all the patients of Amoebic liver abscess who were part of the study. We also Acknowledge the Laboratory department and Radiology Department for their unconditional support.

\section{CONFLICT OF INTEREST}

None.

The Abstract of this full paper was presented at ISID Conference June 2012 at Bangkok, Thailand.

\section{REFERENCES}

1. Stanley SL Jr. Amoebiasis. Lancet 2003;361(9362):1025-34. 
2. Hai AA, Singh A, Miltal VJ, et al. Amoebic liverabscess. Review of 220 cases. Int Surg. 1991;76(2):81-3.

3. Sharma MP, Rai RR. Acharya SK, et al. Needle aspiration of amoebic liverabscess. Br Med J. 1989;299(6711):1308-9.

4. Akgun Y, Tacyildiz IH, Celik Y. Amebic liver abscess: Changing trends over 20 years. World J Surg. 1999;23(1):102-6.

5. Castells L, Porcel JM, Selva A, Ordi J. Amebic liver abscess: Presentation of 4 cases. Rev Esp Enferm Dig 1990;78(6):355-8.

6. Sanjuán FM, Jordá FD, Ginestar JF, Ferrando I, Borghol A, Gutiérrez J. Amebic liver abscess: Medical treatment or percutaneous aspiration? Gastroenterol Hepatol 2007;30(7)399-401.

7. Ananthakrishnan Ramani, Rama Ramani, P G Shivananda. Amoebic liver abscess. A prospective study of 200 casesin a rural referral hospital in south india Bahrain Medical Bulletin. 1995;17(4).

8. Wells, and Miguel Arguedas, Amebic Liver Abscess Southern Medical Journal. 2004;97(7):673-83

9. Yoo HM, Kim WH, Shin SK, Chun WH, Kang JK, Park IS. The Changing Patterns Of Liver Abscess During The Past 20 Yrs A Study Of 482 Cases Yonsei Medical Journal. 1993;34(4):340-51

10. Nari GA, Ceballos Espinosa R, Carrera Ladrón de Guevara S, Preciado Vargas J, Cruz Valenciano JL, Briones Rivas JL, Moreno Hernández F, Góngora Ortega J. Amebic liver abscess. Three years' experience. Rev Esp. Enferm Dig. 2008;100(5):268-72.

11. Seeto RK, Rockey DC. Amebic liver abscess: epidemiology, clinical features, and outcome. West J Med. 1999;170(2):104-9.

12. Shandera WX, Bollam $P$, Hashmey RH, et al. Hepatic amebiasis among patients in a public teaching hospital. South Med J 1998;91(9):829-37.

13. Hoffner RJ, Kilaghbian T, Esekogwu VI, et al. Common presentations of amebic liver abscess. Ann Emerg Med. 1999;34(3):351-5.

14. Kini PM, Mammi MKI. Hepatic amebiasis in Kerala. J Ind Med Assoc. 1970; 55(1):7-10.
15. Joshi VR, Kapoor OP, Purohit $A V$, et al. Jaundice in amoebic abscess of liver. J Assoc Phy India. 1972;20(10):761-4.

16. Khan U, Mirdha BR, Samantaray JC, Sharma MC: Detection of Entamoeba histolytica using polymerase chain reaction in pus samples from amoebic liver abscess. Indian J Gastroenterol. 2006;25(2):55-7.

17. Wiwanitkit V. A note on clinical presentations of amebic liver abscess: an overview from 62 Thai patients, BMC Family Practice. 2002;3(1):1.

18. Sharma et al. BMC Research Notes 2010, 3:21 http://www.biomedcentral. com/1756-0500/3/21.

19. Haque R, Huston CD, Huges M, Houpt E, Petri WA Jr: Amoebiasis: Review Article. New Engl J Med. 2003;348(16):1565-73.

20. Aikat BK, Bhusnurmath SR, Pal AK, Chuttani PN, Dutta DV: Amoebic liver abscess-A clinicopathologic study. Indian J Med Res 1978;67(1987): 381-91.

21. Sharma MP, Ahuja V. Amoebic liver abscess. JIACM. 2003;4(2):107-11.

22. Ribaudo JM and Ochsner A American Journal of Surgery 1973;125(5):570.

23. Nwafo DC and Egbue M.Annals of the Royal College of Surgeons of England. $1981 ; 63(2): 126$.

24. Lyche KD, Jensen WA, Kirsch CM, et al. Pleuropulmonary manifestations of hepatic amebiasis. West J Med 1990;153(3):275-8.

25. Adeyemo A, Aderounmu A. Intrathoracic complications of amoebic liver abscess Journal of the Royal Society of Medicine 1984;77(1);17-21.

26. Tony JC, Martin TK Arch Med Res. Profile of amebic liver abscess. 1992;23(2):249-50.

27. Adams EB, MacLeod IN: Invasive amebiasis: amebic liver abscess and its complications. Medicine. 1977;56(4):325-34

28. Kubitschek KR, Peters J, Nickeson D, Musher DM: Amebiasis presenting as pleuropulmonary disease. West J Med 1985;142(2):203-7.

29. Ibarra-Perez C: Thoracic complications of amebic abscess of the liver a report of 501 cases. Chest 1981,79(6):672-7.

30. Bockus, H L, Gastroenterology, Vol. IV, $3^{\text {rd }}$ Edition, W B Saunders \& Co., Philad., 1976.

Cite this article : Kushwaha Y, Kapil R, Khurana S. A prospective study of one hundred cases of Amoebic liver abscess in a secondary care hospital of Delhi. Int. J. Med. Public Health, 2016; 6(2):84-87. 